\title{
Factors Affecting the College Students' Career Decision-Making Self-Efficacy in China
}

\author{
Yingshan Bao, a , Ning Cui ${ }^{2,3, b}$ \\ ${ }^{1}$ Psychological Health Education and Consultation Center, Dalian University of Technology, Dalian \\ 116024, China \\ ${ }^{2}$ School of Psychology, Northeast Normal University, Changchun 130024, China; \\ ${ }^{3}$ School of Maxism, Changchun University of Chinese Medicine, Changchun 130117, China \\ a157260784@qq.com, bbaoys@dlut.edu.cn
}

Keywords: college students, career decision-making self-efficacy, personality, mental health.

\begin{abstract}
To estimate the overall situation of college students' career decision-making self-efficacy (CDMSE) and the relationship between CDMSE and their personality as well as mental health, the researchers selected and investigated 2880 college students in China. The results showed that: (1) College students, CDMSE was slightly higher than the medium level and their highest score was "information collection" while the lowest is "problem solving". (2) According to the correlation analysis, a significant positive correlation was found between college students' CDMSE and personality as well as mental health. (3) The regression analysis showed that seven variables, which were neuroticism, introversion or extroversion, psychotic, dissimulation, gender, somatization and nationality, were involved in the regression equation and they could be used to explain $20.1 \%$ of variables in CDMSE.
\end{abstract}

\section{Introduction}

At present, Chinese higher education has transformed from former elite education to higher education popularization stage. Since 1999, colleges and universities has started to expand enrollment reaching 1.6 million that year and in 2001 it reached 2.68 million; and it increased to 5.99 million in 2008 and 7 million in 2015. One direct result for higher education popularization was rapid increase of college graduates. According to statistics, college graduates in 2001 around the whole nation was 1.14 million and in 2008, it was 5.59 million then in 2015 it reached 7.49 million, which set new historical high record. As college graduates grew rapidly yet the society's demand for them was limited, it made it even harder for college graduates' employment. In recent years, the economy has grown slowly because of resources shortage, environment deterioration, capital and land supply decrease plus cost increase in manufacturing industry, which made it severer for college graduates' employment. Hence, college graduate's choices of proper occupations have become researchers' universal focus matter. The study found that career decision-making self-efficacy would affect career choices. The graduates with higher career decision-making self-efficacy would take active moves in occupational choices and would venture a wide range of occupations [1]. Compared with individual interest, talents and values, self-efficacy has prominent prediction on individual career choice [2].

\section{Literature Review}

Self-efficacy theory was first brought up by Bandura in 1977 and it refers to individual confidence in reaching their own behavioral objectives. Hackett and Betz originally put self-efficacy into career and proposed the concept of career self-efficacy. Career decision-making self-efficacy means decision makers' assessment and confidence in his/her essential capabilities in completing all tasks during career decision-making [3]. Its operational definition includes confidence in the following five 
specific tasks of abilities: collecting occupational information, formulating career planning, career goal orientation, self-recognition and problem solving.

The previous study indicated that personality and mental health are somehow related to career decision-making. Shang Jain [4] found that proactive personality has prominent prediction role in career decision-making self-efficacy. Many relevant researches identified that anxiety and career decision-making are related [5-7], which would affect the process of career decision-making and recognition gaining [8-9]. Feng Jing et al [10] believed that higher vocational college students with higher career decision-making self-efficacy are more mentally healthier. This paper would apply empirical approach to study the actuality of Chinese college graduates' career decision-making self-efficacy and explore the impacts of personality and mental health on career decision-making self-efficacy, which would benefit to improve college students' occupational psychology quality and provide reference for employment education and psychology education.

\section{Objects and Methods}

\subsection{Research Objects.}

Through random sampling method, a questionnaire was carried out among 2880 students at five colleges and universities in Liaoning Province China and 2762 questionnaires were collected reaching $95.90 \%$. Finally, there were 2680 questionnaires excluding the invalid questionnaire and the validity reached $97.03 \%$. For this questionnaire, there were 664 female students $(24.78 \%)$ and 2016 male students (75.22\%). Among these students, $625(23.32 \%)$ are freshman, $682(25.45 \%)$ are sophomore (23.51\%), 630 are junior and 743 (27.72\%) are senior. And 345(12.87\%) of them are from minority and 2355 are Han people. And 179 (6.48\%) of them are culture and management students and $2501(93.32 \%)$ are science and engineering students.

\subsection{Research Tool.}

Eysenck personality questionnaire (EPQ). EPQ was applied in personality assessment, which was written and edited by British famous psychologist Eysenck. The research introduced the revised version of this questionnaire by Professor Gong Laotian from Chinese Hunan Medical University. This revised scale consists of 88 questions including EPQ-E (E), EPQ-N (N), EPQ-P (P), and EPQ-L. Now this scale has been widely proved to be with good credibility and validity in china.

The symptom checklist-90(SCL-90). SCL-90 covers 90 items including 10 subscales covering Somatization (SOM), Obsessive-Compulsive (O-C), Interpersonal Sensitivity (I-S), Depression (DEP), Anxiety, (ANX), Hostility, (HOS), Phobic Anxiety (PHOB), Paranoid Ideation (PAR), Psychoticism (PSY), and Additional Items(A-I), 5-level assessment is used to scored, the higher the score, the higher contained symptom level; It involves human's feelings, emotions, thinking, consciousness, behavior and living habit, interpersonal relations, diet and sleep. The scale has been proved to have good reliability and validity.

Career decision-making self-efficacy scale (CDMSE).This research of career decision-making self-efficacy introduced the Career Decision-making Self-efficacy Scale[11] made by Pang Yogini and Long Laron (2001), which was revised based on scaled created by Betz and Taylor (1983). This scale is made up of 39 questions covering five factors and they are self-evaluation, information collection, goal selection, plan making, and problem Solution. The scale adopted Liker five points system and the higher the score, the higher the career decision-making self-efficacy. The coefficient of internal consistency is ranging from 0.68 to 0.81 . The coefficient of internal consistency for this scale was 0.94 ; the test-retest reliability of all factors was 0.51 to 0.60 ; the test-retest reliability of the total scale was 0.66 .

\subsection{Statistical Approach.}

The research adopted spas 17.0 to have statistical data analysis. Throughout relevant analysis, it helps to understand relationship among college students' personality, mental health and career decision-making self-efficacy. Based on regression analysis, it explored the impacts of college students' personality, mental health on their career decision-making self-efficacy. 


\section{Results}

\subsection{The Overall Situation of College Graduates' CDMSE.}

According to normality test of college students' career decision-making self-efficacy scores, it found that the data was in normal distribution and its kurtosis value and measure of scenes were under 1. For better observation and understanding, here below are the average scores of items in Table 1.

Table 1. Overall situation of college graduates comes

\begin{tabular}{|c|c|c|c|c|c|}
\hline self-evaluation & information collection & goal selection & plan making & problem Solution & the total of CDMSE \\
\hline $3.86 \pm 0.88$ & $3.93 \pm 0.86$ & $3.87 \pm 0.86$ & $3.75 \pm 0.87$ & $3.71 \pm 0.85$ & $149.41 \pm 32.72$ \\
\hline
\end{tabular}

As shown in Table 1, the average score of college students' CDMSE was 149.41. According to the instruction of points, the original sum of the total scale was 195, which indicated that college students have higher career decision-making self-efficacy. Meanwhile, according to the five-point scale, the average of all factors values is above median; this suggested that college students' career decision-making efficacy were above average. Specifically speaking, college students scored highest in "information collection" and lowest in "problem Solution".

\subsection{Relations between Personality \& Mental Health and CDMSE.}

Make relevant analysis on five factors of career decision-making self-efficacy, four elements of personality and 10 facts of mental health. The result was shown in Table 2.

Table 2. Relations between personality \& mental health and CDMSE

\begin{tabular}{|c|c|c|c|c|c|}
\hline & self-evaluation & information collection & goal selection & plan making & problem Solution \\
\hline EPQ-E & $0.250^{* *}$ & $0.237^{* *}$ & $0.232^{* *}$ & $0.270^{* *}$ & $0.257^{* *}$ \\
\hline EPQ-N & $-0.326^{* *}$ & $-0.314^{* *}$ & $-0.297^{* *}$ & $-0.305^{* *}$ & $-0.291^{* *}$ \\
\hline EPQ-P & $-0.251^{* *}$ & $-0.247^{* *}$ & $-0.210^{* *}$ & $-0.224^{* *}$ & $-0.202^{* *}$ \\
\hline EPQ-L & $0.268^{* *}$ & $0.249^{* *}$ & $0.267^{* *}$ & $0.253^{* *}$ & $0.255^{* *}$ \\
\hline SOM & $-0.257^{* *}$ & $-0.259^{* *}$ & $-0.248^{* *}$ & $-0.250^{* *}$ & $-0.239^{* *}$ \\
\hline O-C & $-0.272^{* *}$ & $-0.265^{* *}$ & $-0.278^{* *}$ & $-0.285^{* *}$ & $-0.282^{* *}$ \\
\hline I-S & $-0.273^{* *}$ & $-0.274^{* *}$ & $-0.274^{* *}$ & $-0.291^{* *}$ & $-0.287^{* *}$ \\
\hline DEP & $-0.288^{* *}$ & $-0.289^{* *}$ & $-0.276^{* *}$ & $-0.294^{* *}$ & $-0.290^{* *}$ \\
\hline ANX & $-0.272^{* *}$ & $-0.270^{* *}$ & $-0.266^{* *}$ & $-0.272^{* *}$ & $-0.271^{* *}$ \\
\hline HOS & $-0.262^{* *}$ & $-0.258^{* *}$ & $-0.246^{* *}$ & $-0.255^{* *}$ & $-0.259^{* *}$ \\
\hline PHOB & $-0.252^{* *}$ & $-0.249^{* *}$ & $-0.240^{* *}$ & $-0.251^{* *}$ & $-0.245^{* *}$ \\
\hline PAR & $-0.241^{* *}$ & $-0.244^{* *}$ & $-0.241^{* *}$ & $-0.252^{* *}$ & $-0.246^{* *}$ \\
\hline PSY & $-0.281^{* *}$ & $-0.277^{* *}$ & $-0.270^{* *}$ & $-0.281^{* *}$ & $-0.277^{* *}$ \\
\hline A-I & $-0.264^{* *}$ & $-0.266^{* *}$ & $-0.253^{* *}$ & $-0.261^{* *}$ & $-0.261^{* *}$ \\
\hline
\end{tabular}

Relevant analysis suggested that college students' CDMSE and mental health facts had negative correlation and it was the same with EPQ-N (N) and EPQ-P (P) in personality. However, it had positive correlation with EPQ-E (E) and EPQ-L (L) in personality.

\subsection{The Prediction Role of Personality and Mental Health on CDMSE.}

The paper conducted regressive analysis taking demography, personality and mental health as independent variables and career decision-making self-efficacy as dependent variables. Details as Table 3.

Table 3. Factors affecting the college students' CDMSE

\begin{tabular}{ccccc}
\hline Model & R 2 & B & Beta & t \\
\hline EPQ-N & 0.098 & -0.407 & -0.194 & $-9.583^{* * *}$ \\
EPQ-E & 0.158 & 0.792 & 0.227 & $12.832^{* * *}$ \\
PSY & 0.179 & -6.139 & -0.099 & $-3.365^{* *}$ \\
EPQ-L & 0.195 & 0.469 & 0.135 & $7.096^{* * *}$ \\
Sex & 0.198 & -4.196 & -0.055 & $-3.153^{* *}$ \\
SOM & 0.200 & -4.638 & -0.067 & $-2.334^{*}$ \\
Nationality & 0.201 & 3.764 & 0.039 & $2.225^{*}$ \\
\hline
\end{tabular}


From the regressive process, it can be seen that seven variables of EPQ-N (N), EPQ-E (E), EPQ-P (P), EPQ-L (L), sex, somatization, and nationality were involved in the regression equation. They could explain and account for $20.1 \%$ in career decision-making self-efficacy.

\section{Conclusion}

\subsection{Status Analysis of College Students' CDMSE.}

The result that college students' CDMSE was above average and that they had highest scores in information collection and lowest in problem solving was in accord with college students' actuality. Currently, colleges and universities paid high values on graduates' employment. And the colleges and universities opened employment education courses widely and hold various occupation events quite often. In addition, as the students are growing ages and they are getting mature. All these conditions allowed college graduates had highe CDMSE.

Specifically speaking, college students' high information collection score and low problem Solution score are mainly because the rapid development of Internet, especially mobile network development, which enabled students to get employment information more easily and conveniently. Thus they scored high in information collection in the career decision-making self-efficacy total scale. However, college students are still in process of study and not entering society yet, they lack social experience and coping capacity when confronting with barriers in career. Therefore, they scored the lowest in problem solving in the career decision-making self-efficacy total scale.

\subsection{Influential Factors Analysis of College Students' CDMSE.}

Based on relevant analysis, college students' CDMSE was prominently connected with personality, mental health. And according to regression analysis, it can be seen that seven variables of EPQ-N (N), EPQ-E (E), EPQ-P (P), EPQ-L (L), sex, somatization, and nationality were involved in the regression equation. They could explain and account for $20.1 \%$ in career decision-making self-efficacy. Factors affecting students' career development can be divided into external environment factor and students' own factors. Usually, it is hard to change the external environment factor. The research indicated us that colleges and universities can help develop students' career decision-making self-efficacy and improve their employment capacity via promoting students' mental health status and perfecting personality.

\section{Acknowledgments}

This work is supported by Social Science Foundation of Liaoning Province of China (L15CSH006); the Fundamental Research Funds for the Central Universities (DUT17RW101) and the Jilin Education Science 13th Five-Year Plan (GS17069)

\section{References}

[1]. D.L. Blustein, The role of goal instability and career self-efficacy in the career exploration process, Journal of Vocational Behavior, vol. 35, no. 2, 1989, pp. 194-203.

[2]. R.W. Lent, et al., Testing social cognitive interest and choice hypotheses across Holland types in Italian high school students., Journal of Vocational Behavior, vol. 62, no. 1, 2003, pp. 101-118.

[3]. K.M. Taylor and N.E. Betz, Applications of self-efficacy theory to the understanding and treatment of career indecision., Journal of Vocational Behavior, vol. 22, no. 1, 1983, pp. 63-81.

[4]. J. Shang, Analysis of the Effects of the Proactive Personality on Graduates' Career Decision-Making Self-Efficacy, Beijing Daxue Xuebao Ziran Kexue Ban/acta Scientiarum Naturalium Universitatis Pekinensis, vol. 45, no. 3, 2009, pp. 548-554.

[5]. S. Folkman and R.S. Lazarus, [An analysis of coping in a middle-aged community sample]., Journal of Health \& Social Behavior, vol. 21, no. 21, 1980, pp. 219-239. 
[6]. D.R. Fuqua and B.W. Hartman, A Behavioral Index of Career Indecision for College Students., Journal of College Student Personnel, vol. 24, no. 6, 1983, pp. 507-512.

[7]. D.R. Fuqua, et al., The relationship of career indecision and anxiety: A multivariate examination, Journal of Vocational Behavior, vol. 30, no. 30, 1987, pp. 175-186.

[8]. K.R. Kelly and W.C. Lee, Mapping the Domain of Career Decision Problems, Journal of Vocational Behavior, vol. 61, no. 2, 2002, pp. 302-326.

[9]. D.L. Blustein and S.D. Phillips, Individual and contextual factors in career exploration, Journal of Vocational Behavior, vol. 33, no. 2, 1988, pp. 203-216.

[10]. J. Feng and H.Y. Liu, An Empirical Study of Pertinency of Career Decision-Making Self-Efficacy Sense and the Situation of Mental-Health of Students in Vocational Colleges, Theory \& Practice of Education, vol. 30, no. 4, 2010, pp. 25-27.

[11]. Y. Peng and L. Long, Study on the Scale of Career Decision-making Self-efficacy for University Students, Chinese Journal of Applied Psychology, vol. 7, no. 2, 2001, pp. 38-43. 